\title{
Expression of the Helicobacter pylori adhesin SabA is controlled via phase variation and the ArsRS signal transduction system
}

Correspondence
Mark H. Forsyth
mhfors@wm.edu

Received 18 December 2007

Revised 1 April 2008

Accepted 8 May 2008

\author{
Andrew C. Goodwin, ${ }^{1} \dagger \ddagger$ Daniel M. Weinberger, ${ }^{1} \dagger \S$ Christopher B. Ford, ${ }^{1} \S$ \\ Jessica C. Nelson, ${ }^{1} \|$ Jonathan D. Snider, ${ }^{1}$ Joshua D. Hall, ${ }^{1}$ \# \\ Catharine I. Paules, ${ }^{1}+\dagger$ Richard M. Peek, $\mathrm{Jr}^{2,3}$ and Mark H. Forsyth ${ }^{1}$ \\ ${ }^{1}$ Department of Biology, The College of William and Mary, Williamsburg, VA 23187-8795, USA \\ ${ }^{2}$ Division of Gastroenterology and Department of Cancer Biology, Vanderbilt University School of \\ Medicine, Nashville, TN 37232-2279, USA \\ ${ }^{3}$ Department of Veterans Affairs Medical Center, Nashville, TN 37212, USA
}

\begin{abstract}
Adaptation to the acidic microenvironment, and adherence to mucosal epithelium, are essential for persistent colonization of the human stomach by Helicobacter pylori. The expression of SabA, an adhesin implicated in the ability of $H$. pylori to adhere to the host gastric epithelium, can be modulated by phase variation via slipped-strand mispairing in repetitive nucleotide tracts located in both the promoter region and the coding region. This study demonstrates the occurrence of phase variation at the sabA locus within individual strains of $H$. pylori, and among multiple isolates from a single patient. In addition, transcription of $s a b A$ is repressed by the acid-responsive ArsRS two-component signal transduction system in vitro. Our results demonstrate that isogenic inactivation of the arsS (jhp0151/HP0165) histidine kinase locus results in a 10-fold SabAdependent increase in adherence to gastric epithelial cells in strain $\mathbf{9 9}$ (contains an in-frame $s a b A$ allele), but not in strain 26695 (out-of-frame $s a b A$ allele). The combination of transcriptional regulation of the sabA locus by the ArsRS two-component signal-transduction system and the generation of subpopulations harbouring alternate $s a b A$ alleles by slipped-strand mispairing during chromosomal replication could permit $H$. pylori to rapidly adapt to varying microenvironments or host immune responses. As a pathogen with a paucity of regulatory proteins, this dual regulation indicates that SabA expression is a tightly regulated process in $H$. pylori infection.
\end{abstract}

†These authors contributed equally to this work.

‡Present address: Graduate Program in Cellular and Molecular Medicine, Johns Hopkins School of Medicine, Baltimore, MD 21205, USA.

§Present address: Department of Immunology and Infectious Diseases, Harvard School of Public Health, Boston, MA 02115, USA.

||Present address: National Institute of Neurological Diseases and Stroke, Bethesda, MD 20892, USA.

-Present address: University of Michigan Medical School, Ann Arbor, MI 48109, USA.

\#Present address: Department of Microbiology and Immunology, The University of North Carolina, Chapel Hill, NC 27599, USA.

†tPresent address: University of Nebraska Medical Center, Omaha, NE 68198, USA.

Abbreviations: AFLP, amplified fragment length polymorphism; CT repeat, cytosine-thymine repeat; FAM, 6-carboxyfluorescein; gDNA, genomic DNA; poly-T, homopolymeric thymine; sialyl-Le ${ }^{x}$, sialyl-dimeric Lewis X; TCST, two-component signal transduction.

\section{INTRODUCTION}

Helicobacter pylori is a Gram-negative bacterium that infects more than half the world's population, and it colonizes the human gastric epithelium. Colonization generally occurs in childhood and, without treatment, persists for the lifetime of the host. As one of the most genetically diverse bacterial species, $H$. pylori has coevolved with human hosts, and generated populations that productively colonize a particular gastric niche. Although many colonized individuals remain asymptomatic, $H$. pylori is a major aetiological agent of peptic ulcer disease, and a recognized risk factor for gastric cancer (Blaser \& Berg, 2001; Merrell \& Falkow, 2004; Kusters et al., 2006).

Adherence to host cell receptors protects $H$. pylori from clearance during mucus shedding, and ensures consistent access to nutrients released by damaged gastric epithelial cells, facilitating long-term colonization, and potentially contributing to disease onset (Gerhard et al., 1999; 
Odenbreit, 2005; Aspholm et al., 2006). H. pylori has several well-characterized adhesins, including BabA, which binds to the Lewis B (Le ${ }^{\mathrm{b}}$ ) antigen (Boren et al., 1993; Ilver et al., 1998), and SabA, which binds to glycosphingolipids displaying a sialyl-dimeric Lewis $\mathrm{X}$ (sialyl-Le ${ }^{\mathrm{x}}$ ) antigen (Mahdavi et al., 2002).

Glycoconjugates bearing the sialyl- $\mathrm{Le}^{\mathrm{x}}$ antigen are rarely expressed in healthy gastric epithelial cells (Madrid et al., 1990), but they are upregulated during inflammation, and serve as binding sites for host cell adhesins of the selectin family (Alper, 2001). Accordingly, SabA-mediated adherence is positively correlated with sialyl- $\mathrm{Le}^{\mathrm{x}}$ concentration in vitro (Linden et al., 2004), and colonization density is increased in patients who produce high levels of sialyl-Le ${ }^{\mathrm{x}}$, or are infected with SabA-positive strains of $H$. pylori (Sheu et al., 2006). H. pylori shows a tropism for areas of reduced acidity in the stomach that contain gastric pit cells producing sialyl-Le ${ }^{\mathrm{x}}$ (Bjorkholm \& Salama, 2003), and studies have shown that clearance of infection reduces production of sialyl- $\mathrm{Le}^{\mathrm{x}}$ receptors to pre-infection levels (Mahdavi et al., 2002; Acheson \& Luccioli, 2004; Roche et al., 2004).

Expression of bacterial adhesins can be regulated both by reacting to changes in the environment using signal transduction, and by generating genetic changes that affect production of functional proteins. It has been demonstrated that some $H$. pylori genes are regulated by phase variation (Saunders et al., 1998; Salaun et al., 2004), a mechanism by which genes can be expressed in an all-ornothing manner. Phase variation can control gene expression at the transcriptional and the translational levels, and in other organisms it has been shown to mediate evasion of the host immune response, or to modify virulence properties (van der Woude \& Baumler, 2004). At the level of chromosomal replication, a molecular mechanism of phase variation known as slipped-strand mispairing can insert or delete nucleotides within repetitive DNA tracts, usually near the $5^{\prime}$ end of genes. This results in altered reading frames, alternatively yielding truncated or full-length proteins (de Vries et al., 2002).

The sabA locus contains a homopolymeric thymine (poly$\mathrm{T})$ tract in the promoter region, and a dinucleotide cytosine-thymine repeat (CT repeat) in the coding region. Studies have demonstrated that collections of $H$. pylori strains exhibit significant diversity in the presence of $s a b A$, CT-repeat tract lengths, and resulting expression of SabA (Lehours et al., 2004; Sheu et al., 2006; Yamaoka et al., 2006). This regulatory mechanism may explain why $1 \%$ of J99 colonies in one study spontaneously lost their ability to bind sialyl-Le ${ }^{\mathrm{x}}$ (Mahdavi et al., 2002). Yamaoka et al. (2002) demonstrated that adherence, colonization ability, bacterial density, and induction of inflammation were all decreased when $s a b A$ or $s a b B$ was switched off, indicating that this mechanism of $s a b A$ regulation has functional significance.

Aside from genetic changes, H. pylori also uses twocomponent signal transduction (TCST) systems to respond to environmental changes. Activation of a TCST system results in changes in the rates of histidine kinase and response regulator protein phosphorylation, leading to altered promoter-region DNA-binding activity of the response regulator, and either positive or negative regulation of gene transcription (Beier \& Frank, 2000; Stock et al., 2000). A previous study in our laboratory utilized DNA macroarrays to define the set of genes regulated by the HP0165-HP0166 TCST system in Helicobacter pylori strain 26695 (Forsyth et al., 2002). That study, which compared genome-wide transcriptional profiles between wild-type $H$. pylori and an isogenic HP0165 histidine kinase mutant, found one gene to be repressed in the null mutant, while six genes, including sabA (HP0725), were derepressed in the mutant.

Additional studies have further characterized and expanded this regulon, and identified acidic $\mathrm{pH}$ as the key environmental signal for HP0165-HP0166, and this locus has thus been redesignated arsRS (acid-responsive signalling) (Dietz et al., 2002; Pflock et al., 2004; Sachs et al., 2006). arsRS has accordingly been demonstrated to be essential for the production of urease under acidic conditions (Panthel et al., 2003), and is required for virulence in a mouse model (Pflock et al., 2005). Transcription of the response regulator locus arsR (HP0166), an essential gene in H. pylori (Beier \& Frank, 2000), is downregulated at pH 5.0 (Bury-Mone et al., 2004) and non-phosphorylated ArsR has additional, phosphorylation-independent, regulatory activity (Schar et al., 2005).

Recent studies have provided further insights into the relationship between $\mathrm{pH}$ and the expression of the genes regulated by ArsRS at the transcriptional and translational levels. Global gene expression analyses by Merrell et al. (2003) and Bury-Mone et al. (2004) have found that at pH $5.0 \mathrm{sabA}$ and its paralogue $s a b B$ (de Jonge et al., 2004) are downregulated, while HP1188, a novel $H$. pylori adhesin (Rubinsztein-Dunlop et al., 2005), is induced. It has also been reported that SabA-positive status is associated with decreased acid secretion in patients, and that SabA protein levels are reduced at pH 5.0 (Yamaoka et al., 2006).

In the present study, we hypothesized that the role of SabA in $H$. pylori adherence to AGS gastric epithelial cells is governed by phase variation and transcriptional regulation of sabA via the ArsRS system. We demonstrate that derepression of $s a b A$ transcription in an ArsS isogenic knockout strain of $H$. pylori (Forsyth et al., 2002) results in a corresponding functional change in the ability of the bacterium to adhere to gastric epithelial cells. In addition, we demonstrate the existence of multiple alleles of sabA within a single $H$. pylori strain population, and among multiple isolates from a single patient, differing in the nucleotide-repeat tract lengths. Our findings provide new insights into the complex mechanisms regulating the expression of the SabA adhesin and may contribute to an improved understanding of persistent $H$. pylori infection, and thus have implications for development of therapeutics. 


\section{METHODS}

Bacterial culture. The $H$. pylori strains used in this study are described in Table 1. H. pylori was cultured on Trypticase Soy Agar II plates with $5 \%$ sheep blood (BBL) at $37{ }^{\circ} \mathrm{C}$ and $5 \% \mathrm{CO}_{2}$, or Brucella agar plates supplemented with $10 \%$ newborn calf serum (Gibco/ Invitrogen). Escherichia coli $\mathrm{DH} 5 \alpha$ was cultured in Luria-Bertani medium. When appropriate, media were supplemented with $100 \mu \mathrm{g}$ ampicillin $\mathrm{ml}^{-1}, 20 \mu \mathrm{g}$ kanamycin $\mathrm{ml}^{-1}, 25 \mu \mathrm{g}$ chloramphenicol $\mathrm{ml}^{-1}$ (for E. coli), or $5 \mu \mathrm{g}$ chloramphenicol $\mathrm{ml}^{-1}$ (for H. pylori).

Amplified fragment length polymorphism (AFLP) analysis. Oligonucleotides were designed to amplify the regions containing the poly-T (sabAampF and sabArev; see Table 2 for oligonucleotide sequences) and CT-repeat (sabAForC and HP0725R) tracts, as well as a downstream control region (sabABCFw and sabABCon). A 6carboxyfluorescein (FAM) label was added to the $5^{\prime}$ end of one primer from each oligonucleotide pair. PCRs for AFLP analysis were performed using Vent High-Fidelity DNA polymerase (New England Biolabs). Amplicons were purified (QiaQuick PCR purification kit; Qiagen), processed at the DNA Sequencing and Synthesis Facility (Iowa State University, Ames, IA, USA), and analysed using GENESCAN software (Applied Biosystems).

Sequencing of sabA clones. The $s a b A$ poly-T region was amplified by PCR from $H$. pylori strains 26695 and $J 99$ with primers sabAampF and sabArev. A population of clones (denoted as pSabA-T) was generated by cloning the resulting PCR products in pGEM-T Easy. Similarly, the $s a b A$ CT-repeat tract region was amplified from $H$. pylori 26695 genomic DNA (gDNA) or cDNA with primers HP0725ForwII and HP0725Ext, and cloned to create a collection of clones denoted as pSabA-gDNA-CT and pSabA-cDNA-CT, respectively. Plasmid DNA was isolated using the QiaSpin Miniprep kit (Qiagen) or Wizard Plus Midiprep kit (Promega), and clones were sequenced as described below to determine the length of the poly-T or CT-repeat tracts.

DNA sequencing. Sequencing reactions were performed using the Big Dye v3.1 system (Applied Biosystems), purified over DTR gelfiltration spin columns (Edge Biosystems), vacuum-dried, and resuspended in Hi-Di Formamide (Applied Biosystems). Denatured samples were sequenced on an ABI 3100 Avant (Applied Biosystems), and analysed with Sequencing Analysis 5.1.1 (Applied Biosystems) and MacVector 7.0 (MacVector) software.

Quantitative real-time PCR. RNA was extracted from exponentialphase H. pylori cultures using the Invitrogen RNA Extraction System, treated with Turbo DNase (Ambion), and assayed for gDNA contamination by PCR. cDNA was synthesized from $2 \mu \mathrm{g}$ RNA using random hexamers (Applied Biosystems) and AMV reverse transcriptase (Promega), purified using the QIAquick Nucleotide Removal kit (Qiagen), and verified via PCR. Quantitative real-time PCR was performed using the iCycler iQ real-time PCR detection system and SYBR Green supermix reagents (Bio-Rad). Relative expression of sabA (primers HP0725fwd and HP0725rev), HP0218 (control gene; primers HP0218forw and HP0218rev) and gyrB (normalization gene; primers gyrB forw and gyrB rev) was calculated for J99 and the J99ars $:$ : : cat mutant strain. PCRs were performed in triplicate, and meltcurve analysis was used to ensure that a single product was amplified with each primer set. Differences in gene expression from three independent experiments were calculated by the $\Delta \Delta C_{\mathrm{T}}$ method (Livak \& Schmittgen, 2001), and evaluated by Student's $t$-test.

Creation of $H$. pylori sabA : : cat, arsS : : cat, arsS : : $k m$ and arsS complemented mutants. A 525 bp fragment was amplified by PCR from the $5^{\prime}$ region of $H$. pylori $\mathrm{J} 99$ sabA using primers JCN725F and JCN725R, and cloned into pGEM-T Easy (Promega) to generate pJCN1. The chloramphenicol acetyltransferase gene (cat) was excised from pCM7 (a kind gift of Dr John Loh and Dr Timothy Cover, Vanderbilt University Medical Center, Nashville, TN, USA) and cloned into the pJCN1 sabA HindIII site, resulting in pJCN2. H. pylori strain J99 was naturally transformed with pJCN2, as previously described (Forsyth et al., 2002), yielding strain J99-sabA : : cat. The cat cassette was also inserted into the arsS BglII site to generate strain J99-arsS : : cat.

H. pylori strains J99-165Km, J99-165Km-WT and J99-165Km-vector were a kind gift of Dr John Loh and Dr Timothy Cover (Loh \& Cover, 2006). The ars $S:: \mathrm{km}$ mutant allele from $\mathrm{J} 99-165 \mathrm{Km}$ was naturally transformed into passage-level-matched strains J99 and J99-sabA::cat to generate strains J99-ars $S:: \mathrm{km}$ and J99-arsS : $\mathrm{km}$-sabA : : cat used in this study. Strain J99-ars $S:: k m-r d x A:: a r s S$, containing a complemented ars $S$ allele, was constructed by natural transformation of the $r d x A:: a r s S$ allele from $\mathrm{J} 99-165 \mathrm{Km}-\mathrm{WT}$ into J99-ars $S: \mathrm{km}$, and selection on medium containing $15 \mu \mathrm{g}$ metronidazole $\mathrm{ml}^{-1}$. The ars : : cat mutant in a strain 26695 background has been described previously (Forsyth et al., 2002).

H. pylori adhesion to AGS cells. The AGS (human gastric epithelial cell adenocarcinoma; ATCC) cell line was cultured in F12 Kaighn's medium supplemented with $10 \%$ fetal bovine serum (Gibco/ Invitrogen) at $37{ }^{\circ} \mathrm{C}$ and $5 \% \mathrm{CO}_{2}$. The cells were then used to seed 24-well plates with $2 \times 10^{5}$ cells per well, and the plates were maintained at $37{ }^{\circ} \mathrm{C}$ and $5 \% \mathrm{CO}_{2}$ overnight. Plate-grown $H$. pylori was resuspended in $3.5 \mathrm{ml} \mathrm{F} 12 / 10 \%$ FBS, concentration was

Table 1. $H$. pylori strains used in this study

\begin{tabular}{|c|c|c|}
\hline Strain & Description & Reference \\
\hline $\mathrm{J} 99-a r s S:: \mathrm{km}$ & arsS locus disrupted by kanamycin-resistance cassette & Loh \& Cover (2006) \\
\hline J99-arsS: : km-sabA::cat & $\begin{array}{l}\text { J99-ars } S:: k m \text { with } s a b A \text { locus disrupted by chloramphenicol-resistance } \\
\text { cassette }\end{array}$ & This study \\
\hline J99-ars $S:: k m-r d x A:: a r s S$ & $\begin{array}{l}\text { J99-ars } S:: k m \text { complemented by insertion of ars } S \text { at the } r d x A \text { locus, } \\
\text { conferring metronidazole resistance }\end{array}$ & Loh \& Cover (2006) \\
\hline J99-ars $S:: k m-r d x A:$ control & $\begin{array}{l}\text { J99-ars } S:: k m \text { with control DNA inserted at } r d x A \text { locus, conferring } \\
\text { metronidazole resistance }\end{array}$ & Loh \& Cover (2006) \\
\hline J99 C-6 & Clinical reisolate from corpus biopsy 6 years after isolation of strain $J 99$ & Israel et al. (2001) \\
\hline 26695 & Clinical isolate, $\operatorname{cagA}$-positive & Tomb et al. (1997) \\
\hline 26695-arsS: : cat & arsS locus disrupted by chloramphenicol-resistance cassette & Forsyth et al. (2002) \\
\hline
\end{tabular}


Table 2. Oligonucleotides used in this study

\begin{tabular}{|ll|}
\hline Name & \multicolumn{1}{c|}{ Sequence $\left(\mathbf{5}^{\prime} \mathbf{- 3}^{\prime}\right)$} \\
\hline HP0218forw & GAATTGACCCCTTGAACAATCC \\
HP0218rev & CGCTAAATTTGGCGGTAACGCTCC \\
HP0725fwd & GGAACATTTTATGAAAAAGACAATTCTG \\
HP0725Rev & TGGCTTAATTGCTCGTATTTTTCG \\
gyrB forw & CGTGGATAACGCTGTAGATGAGAGC \\
gyrB rev & GGGATTTTTCCGTGGGGTG \\
JCN725F & CCAATCAATTTATAGTAAAATTAGGTTCATTG \\
JCN725R & GGAGGGTTACCCTGGACACTAGCGG \\
sabAampF & FAM-GGCTCTAGCAATGTGTGGCAGC* \\
sabArev & AGAATTGTCTTTTTCATAAAATGTTCC \\
sabAforC & FAM-GAAATCCAATCAATTTATAGTAAAATTAGG \\
HP0725R & CGCCGATTTGATAGCCGGCGCTCAC \\
sabABCFw & ATACCCATTTTGCAACAAGCGGTTACGC \\
sabABCon & FAM-TAGGCGTTGAACCCGCATTGGG* \\
HP0725F & CCAATCAATTTATAGTAAAATTAGGTTCATTG \\
HP0725ForwII & GGAACATTTTATGAAAAAGACAATTCTG \\
HP0725Ext & GCTTCAACGAAGCCACTTGATT \\
HP0725Forward & TTTGGCTCTAGCAATGTGTGGC \\
HP0725Reverse & GGGTTTGATGTTGAGTCTGTTGC \\
\end{tabular}

${ }^{\star}$ These oligonucleotides were labelled at the $5^{\prime}$ end with FAM for AFLP analysis. Unlabelled versions of these primers were used for other applications.

estimated by $\mathrm{OD}_{600}$, and the suspension was added to cells at $5 \times 10^{7}$ c.f.u. per well. Plates were then centrifuged at $480 \mathrm{~g}$ for $5 \mathrm{~min}$ to initiate contact between $H$. pylori and AGS cells, and incubated for $3 \mathrm{~h}$ at $37^{\circ} \mathrm{C}$. After incubation, cell monolayers were washed three times with cold $1 \times \mathrm{PBS}(\mathrm{pH} 7.0)$ to remove non-adherent bacteria, and $0.5 \mathrm{ml} \mathrm{F} 12 / 10 \% \mathrm{FBS} / 1 \%$ saponin was added to lyse cells. Tenfold serial dilutions of these lysates were plated on blood agar plates and incubated at $37^{\circ} \mathrm{C} / 5 \% \mathrm{CO}_{2}$ for 4 days, and titres of the original and post-infection cultures were determined. Relative differences in adherence between strains in multiple independent experiments were evaluated by using Student's $t$-test.

\section{RESULTS AND DISCUSSION}

\section{Multiple alleles of sabA exist in H. pylori 26695 and $J 99$ populations due to poly-CT and poly-T tract length variation}

The first annotated $H$. pylori genome (strain 26695) identified the HP0725 locus (subsequently named sabA) as containing putatively phase-variable repetitive nucleotide sequences (Tomb et al., 1997). Depending on the length of a CT-repeat tract near the $5^{\prime}$ end of the sabA ORF, subsequent translation either encounters a premature termination codon (resulting in a truncated, non-functional gene product), or encodes a full-length functional SabA adhesin protein. Strain 26695 is predicted to contain a 14-base poly- $\mathrm{T}$ tract in the promoter region, and to encode an out-of-frame sabA allele containing six CT repeats (see schematic diagram, Fig. 1a). A recent study showed that $49 \%$ of strains examined $(n=89)$ were predicted to be in-frame based on the number of CT repeats (de Jonge et al., 2004). However, to our knowledge, no studies have evaluated the degree of diversity at the $s a b A$ repetitive tracts within a single strain of $H$. pylori, or among multiple strains isolated from a single patient.

We first tested the hypothesis that phase variation could result in $H$. pylori populations containing multiple alleles at the sabA poly-T and CT-repeat tracts. Preliminary evidence that the length of the sabA CT-repeat region varies in strain 26695 was obtained by electrophoresis of PCR products of the repeat-containing region of $s a b A$ on a denaturing polyacrylamide gel. When the region was amplified from a plasmid template (pSabA), a single amplicon was observed, while the same amplification using H. pylori strain 26695 gDNA as template revealed the presence of a second visible band (data not shown). Next, to better demonstrate allelic variation in the poly-T and CT-repeat tracts of the sabA locus, we conducted AFLP analysis. Results of these analyses indicated the presence of alleles of multiple lengths in amplicons containing the sabA poly-T and CTrepeat tracts from strain 26695 (Fig. 1). Control amplicons consisting of a portion of the $s a b A$ locus lacking a repetitive sequence produced a single detectable allele. Analysis of the putatively variable regions amplified from a cloned fragment of $s a b A, \mathrm{pSabA}$, also revealed a single fragment length (data not shown), thus indicating that the variation seen in AFLP analysis was not an artefact of PCR.

To quantify allelic variation at the CT-repeat tract within the sabA coding region of $H$. pylori strain 26695, we 


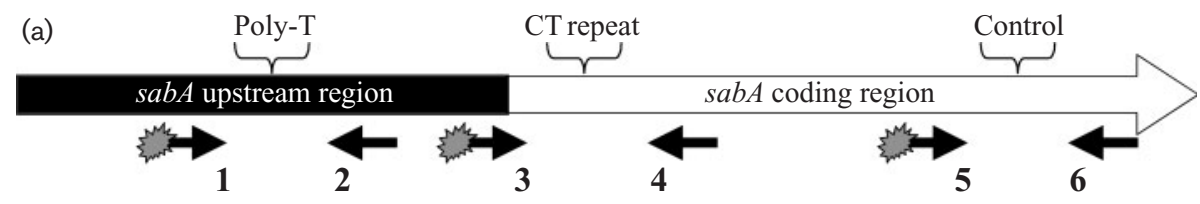

FAM-labelled oligonucleotide

(b)
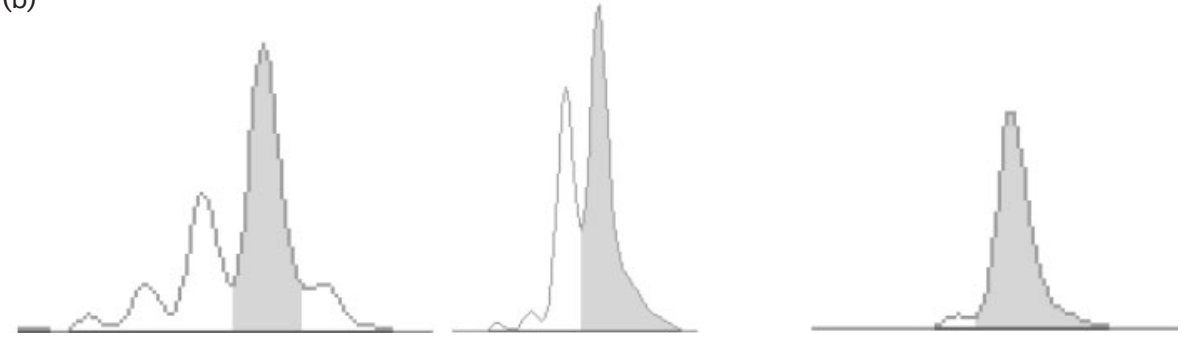

Fig. 1. The H. pylori strain 26695 population in vitro possesses various sabA alleles differing in repetitive sequence lengths. (a) Schematic map of the sabA locus of $H$. pylori 26695 showing the locations of the promoter region poly-T and coding region CT-repeat tracts, as well as a downstream control region lacking any such putatively hypermutable sequences. The relative locations of oligonucleotide pairs used for AFLP analyses are denoted with black arrows. As indicated, one primer of each pair was $5^{\prime}$ labelled with FAM. (b) Fragment length polymorphism traces indicate five size variants in amplicons of the poly-T region, and four size variants in the amplicons of the CT-repeat tract. The predominant polymorphic form is shaded in grey. A single amplicon length was detected in the downstream control region. This region possesses no repetitive sequences.

sequenced pSabA-gDNA-CT clones (Fig. 2a, black bars). Our sequencing results indicated that while $97.6 \%$ (40/41) of the clones confirmed the out-of-frame sabA status, $25 \%$ of these clones contained a tract length of eight CT repeats rather than the predicted length of six repeats. A single clone possessed a sabA allele containing seven CT repeats; this CT tract length is predicted to express a functional SabA adhesin. These data support the hypothesis that slipped-strand mispairing could generate allelic variation at the $s a b A$ locus; subsequent environmental pressures could rapidly select for a subpopulation that does or does not express the SabA adhesin.

We next hypothesized that RNA polymerase could err during transcription instead of, or in addition to, mutations induced by slippage of DNA polymerase during replication. As a result of the coupling of transcription and translation in prokaryotes, such an event could have a significant impact on protein expression. This might allow for the potential synthesis of a full-length mRNA transcript, despite the presence of a gDNA sequence that would predict abortive translation. To investigate this possibility, we cloned and sequenced PCR products amplified from $H$. pylori 26695 cDNA synthesized using a $s a b A$-specific oligonucleotide (pSabA-cDNA-CT; Fig. 2a, grey bars). As with those derived from a gDNA template, nearly all pSabA-cDNA-CT clones analysed $(n=23)$ harboured out-of-frame sabA sequences: $91.3 \%$ contained six or eight CT repeats, while two clones possessed an inframe CT tract with seven repeats. No statistically significant difference in the distribution of $s a b A$ CT-repeat tract lengths was observed in clones derived from cDNA versus gDNA templates ( 2 d.f., $\chi^{2}=1.76, P=0.415$ ). Thus, it seems unlikely that significant expression of SabA could result from the generation of in-frame mRNA transcripts with different CT-repeat tract lengths than the corresponding chromosomal sequence.

Variation in the sabA promoter-region poly- $\mathrm{T}$ tract was likewise characterized by sequencing pSabA-T clones containing amplicons derived from $H$. pylori 26695 or J99 gDNA ( $n=19$ and $n=15$, respectively; Fig. $2 \mathrm{~b}$ ). Sequencing analysis of all clones containing sabA promoter region PCR amplicons from strain 26695 revealed a distribution of 13-16 thymine nucleotides, with a predominant length of 15 bases ( $68 \%$ of clones). pSabA-T clones derived from strain J99 contained 16-19 thymine nucleotides; $40 \%$ of these had a repeat tract length of 17 bases. Annotated genomic sequences indicate poly- $T$ tracts of $14 \mathrm{nt}$ in 26695 (Tomb et al., 1997), and $18 \mathrm{nt}$ in $\mathrm{J} 99$ (Alm et al., 1999). These results provide strong evidence that the $s a b A$ promoter poly- $T$ tract undergoes slippedstrand mispairing, resulting in a population of $H$. pylori with numerous alleles. Further studies will be needed to ascertain the potential effects of poly-T length variation on transcriptional control at the sabA locus. 

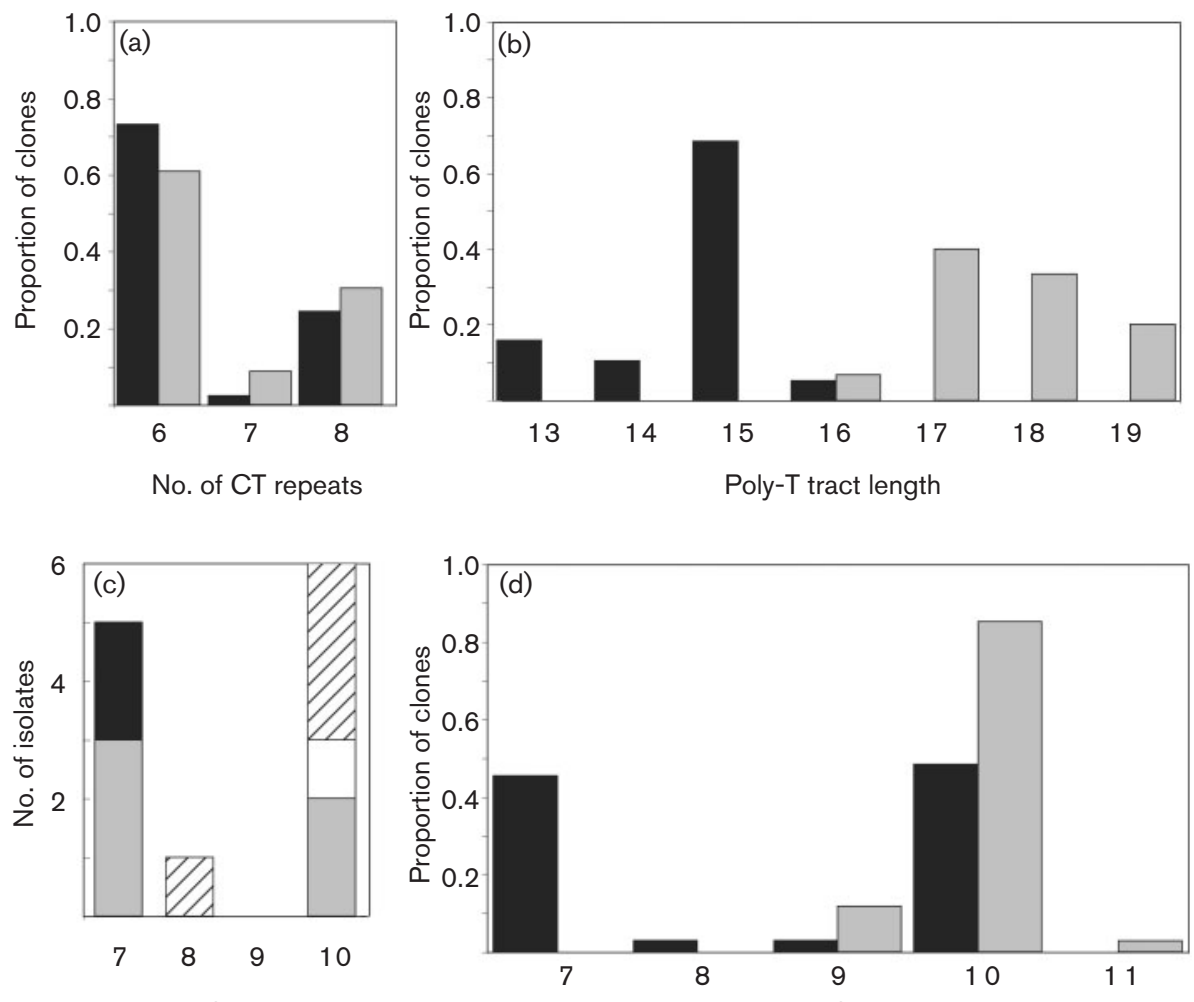

No. of CT repeats

No. of CT repeats

Fig. 2. Allelic diversity in poly- $T$ and $C T$-repeat tracts associated with the sabA locus. The poly- $T$ tract is located in the presumed $s a b A$ promoter region, while the CT-repeat tract lies in the $5^{\prime}$ region of the $s a b A$ coding sequence. In-frame sabA alleles correspond to a dinucleotide tract length of 7 or 10 CT repeats. (a) Distribution of CT-repeat tract lengths in clones amplified from $H$. pylori strain 26695 gDNA (black bars, $n=41$ ) and cDNA (grey bars, $n=23$ ). The published strain 26695 genome sequence predicts a tract of six CT repeats (Tomb et al., 1997). (b) Distribution of poly-T tract lengths in PCR amplicons cloned from $H$. pylori 26695 (black bars, $n=19$ ) and $J 99$ (grey bars, $n=15$ ). (c) Distribution of CT-repeat tract lengths in $\mathrm{J} 99$ re-isolate strains from the antrum (grey bars, $n=5$ ), cardia (white bar, $n=1$ ), corpus (hatched bars, $n=4$ ) and duodenum (black bars, $n=2$ ). (d) Distribution of CT-repeat tract lengths in clones amplified from low (black bars, $n=33$ ) or high in vitro passage ( $>50$ passages, grey bars, $n=34) \mathrm{H}$. pylori J99-C6 (re-isolate from corpus biopsy).

\section{Allelic diversity at the sabA locus exists within a single patient}

In order to gain a better understanding of the actual $s a b A$ diversity within a single host, we analysed a collection of 12 low-passage strains re-isolated by gastric biopsy from the $J 99$ source patient 6 years after the initial endoscopy (Israel et al., 2001). These strains were isolated from several regions of the stomach: antrum $(n=5)$, cardia $(n=1)$, corpus $(n=4)$, and foci of gastric metaplasia in the duodenum $(n=2)$. The $s a b A$ CT-repeat region of each isolate was amplified by PCR and directly sequenced without cloning in order to assess the presence or absence of variation in the length of the tract relative to the archival strain $\mathrm{J99}$ (Fig. 2c). Five isolates had a tract of seven CT repeats (in-frame sabA allele), one isolate contained eight CT repeats (out-of-frame), and six isolates possessed $10 \mathrm{CT}$ repeats (in-frame).

While the annotated genome sequence of archival strain J99 predicts an out-of-frame $s a b A$ locus with nine CT repeats (Alm et al., 1999), 11 of the 12 re-isolates analysed were predicted to be in-frame based on a CT-repeat tract length of 7 or 10 repeats This suggested the possibility that a selective pressure favouring expression of SabA may have developed in the host that was not present when the strain was originally isolated. However, we proceeded to sequence the repetitive region of archival J99, as well as two singlecolony isolates derived from that strain, and found $10 \mathrm{CT}$ repeats, corresponding to a phase-on $s a b A$ allele in each case. Taken together, these sequencing results, along with published observations that J99 (but not 26695) binds the Lewis X antigen (Mahdavi et al., 2002), and adherence data in the current study (see below), suggest that J99 does in fact harbour an in-frame sabA locus. It is possible that a portion of the sabA variation observed in the re-isolates examined in the current study was present at the time strain J99 was initially isolated from an antral biopsy, but not reflected in the published genome sequence. However, as a recent study would suggest (Kuipers et al., 2000), 
selective pressures in the 6 years between the isolation of the original and novel 599 strains probably contributed to further genetic diversity at $s a b A$ and other loci.

One isolate (J99 C-6) was selected for additional study of variation in the CT-repeat tract as described above (Fig. 2d). Plasmid clones $(n=33)$ were generated from the amplicons generated from the sabA CT-repeat region of $H$. pylori 599 C-6, and sequenced to determine the initial diversity present at the sabA locus at the time of the biopsy ('low passage'). In addition, the effect of prolonged in vitro passage on the sabA CT-repeat tract was studied in a similar manner using 34 plasmid clones obtained from PCR amplicons of the same region of $s a b A$ after numerous in vitro passages ('high passage', $>50$ passages). In each case, the vast majority of clones ( $94 \%$ of low-passage and $85 \%$ of high-passage clones; Fisher's exact test, $P=0.43$ ) contained in-frame $s a b A$ alleles with 7 or 10 CT repeats. However, a significant shift towards a longer CT-repeat tract was observed in high-passage clones $\left(\chi^{2}\right.$ for trend $=19.7$, d.f. $=1, P<0.0001)$. These results indicate that a functional SabA was favoured in the gastric niche from which the strain was isolated.

Taken together, the above results clearly demonstrate that phase variation via slipped-strand mispairing occurs at the H. pylori sabA locus, and results in the generation of diversity in the length of promoter region poly- $\mathrm{T}$ and coding region CT-repeat tracts within a single strain, and among multiple isolates from a single patient. As a result of variations in this repetitive nucleotide tract, populations of H. pylori may or may not express the functional SabA adhesin molecules needed to mediate BabA-independent binding to sialyl-Le $\mathrm{e}^{\mathrm{x}}$ antigens on the host cell surface.

\section{Sensory histidine kinase arsS null mutants express a sabA-dependent hyper-adherent phenotype}

We next sought to study the effects of ArsRS-mediated transcriptional control of $s a b A$ on adherence by $H$. pylori strains harbouring in-frame (J99) and out-of-frame (26695) sabA loci. Several studies have demonstrated that the ArsRS TCST system, in response to environmental changes in $\mathrm{pH}$, regulates the transcription of sabA (Dietz et al., 2002; Forsyth et al., 2002; Pflock et al., 2004). We conducted a series of in vitro assays that quantified adherence of $H$. pylori strains to AGS cells to test the hypothesis that deletion of the arsS histidine kinase locus would result in derepression of $s a b A$ transcription and, in strains containing in-frame sabA CT-repeat tracts, increased adherence to gastric epithelial cells.

We first confirmed the transcriptional control of $s a b A$ by arsS (previously demonstrated only by genome-wide transcriptional profiling) by performing quantitative realtime PCR to compare sabA transcription in wild-type $H$. pylori and an isogenic ars $S$ mutant strain (Fig. 3). Results showed a $3.75 \pm 0.25$ fold increase in sabA cDNA in $H$.

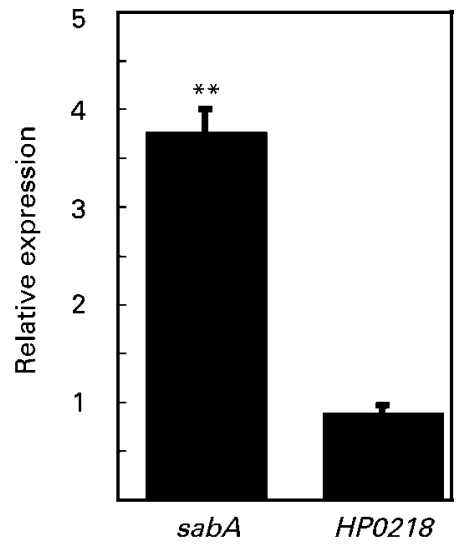

Fig. 3. Derepression of $s a b A$ in J99-arsS::cat mutant. Quantitative real-time PCR analysis was performed using cDNA prepared from wild-type $H$. pylori strain $\mathrm{J} 99$ and the J99-arsS : : cat histidine kinase null mutant. Expression levels (mean \pm SEM) of sabA and HP0218 (arsS-independent control) relative to $\mathrm{J99}$ wildtype from three independent experiments, each performed in triplicate and normalized to $g y r B$, are presented. Asterisks $\left({ }^{\star \star}\right)$ denote significantly higher sabA expression in J99-arsS: : cat compared to wild-type J99 ( $P=0.0026$, Student's $t$-test). No significant difference in HP0218 expression was detected between the two strains ( $P=0.62$, Student's $t$-test).

pylori J99-arsS::cat compared to wild-type $\mathrm{J} 99$ (mean \pm SEM from three independent experiments; $P=0.0026$, Student's $t$ test). These results are concordant with our earlier DNA macroarray study that indicated that the adhesin sabA is 3.34-fold derepressed in the absence of a functional allele of the ArsS histidine kinase (Forsyth et al., 2002). Expression of HP0218, a gene not under the transcriptional control of the ArsRS TCST system, was not significantly different in $\mathrm{J} 99$ versus J99-arsS: : cat $(P=0.62$, Student's $t$ test).

To examine the functional effect of increased transcription of $s a b A$ in the absence of arsS, we assayed the ability of wild-type $H$. pylori strain $\mathrm{J99}$ and three J99-derived mutant strains (J99-ars $S: \mathrm{km}$, J99-ars $S: \mathrm{km}$-sabA::cat and J99ars $S:: \mathrm{km}-r d x A::$ ars $S$ ) to bind to AGS cells in vitro (Fig. 4). Adherence of J99-arsS:: $\mathrm{km}$ to AGS cells was 10.5-fold greater than that of wild-type $J 99\left[1.2( \pm 0.3) \times 10^{7}\right.$ vs $1.1( \pm 0.3) \times 10^{6}$ c.f.u. per well, $P=0.01, n=3$ independent experiments conducted in triplicate]. This increased adherence was reversed when strain J99-arsS:: km was further modified to either inactivate the sabA locus [strain J99-arsS:: km-sabA::cat, $1.1( \pm 0.4) \times 10^{6}$ c.f.u. per well; J99-arsS:: km: $1.2 \pm 0.3 \times 10^{7}$ c.f.u. per well, $n=3$ ] or reintroduce the ars $S$ gene [strain J99-ars $S: \mathrm{km}$ $r d x A:: \operatorname{arsS}, 1.3( \pm 0.6) \times 10^{6}$ c.f.u. per well, J99-ars : : km: $1.2 \pm 0.3 \times 10^{7}$ c.f.u. per well,; $n=2$ ]. No significant difference in mean adherence was detected among J99, J99-arsS:: km-sabA::cat and J99-arsS::km-rdxA::arsS ( $P>0.75$ for all comparisons). 


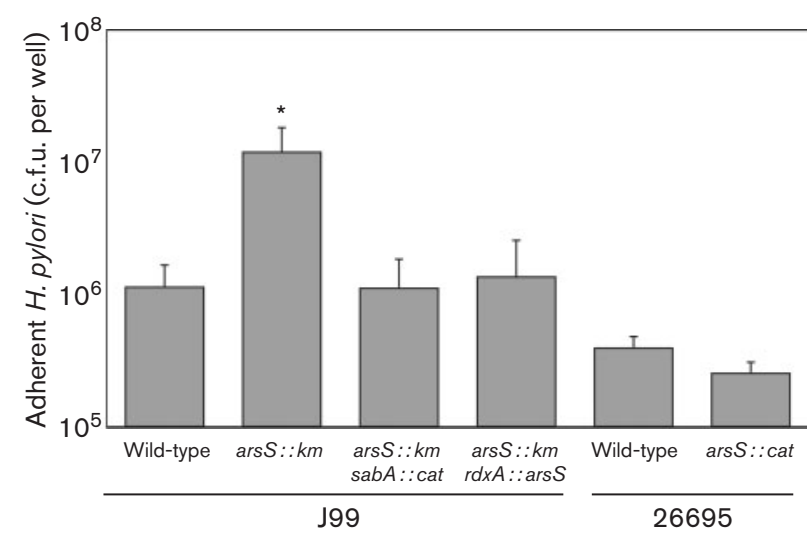

Fig. 4. Role of ArsS in $H$. pylori adherence to AGS cells and the function of $S a b A$ in augmenting adherence. Disruption of ars $S$ (strain J99-ars $S: \mathrm{km}$ ) results in increased adherence of $H$. pylori J99 to AGS cells ( ${ }^{*}$ denotes $P=0.01$ vs wild-type, $n=3$ experiments). The increased binding is abrogated by mutation of the sabA locus (strain J99-arsS : : km-sabA : : cat; $P=0.97$ vs wildtype, $n=3$ experiments) or complementation of the ars $S$ locus (strain J99-arsS::km-rdxA::arsS; $P=0.75$ vs wild-type, $n=2$ experiments). In contrast, disruption of ars $S$ in strain 26695, which contains an out-of-frame sabA allele, does not result in increased binding. Adherence (c.f.u. per well) data from independent experiments performed in triplicate are presented (mean \pm SEM). Differences between strains in mean adherence were evaluated by using Student's $t$-test.

Strain J99-ars $S: \mathrm{km}-r d x A$ : : control, a control strain created by inactivating the $r d x A$ gene with an unrelated sequence (Loh \& Cover, 2006), retained the hyper-adherent phenotype (data not shown), confirming that the decreased binding seen in strain J99-ars $S:: k m-r d x A$ : : ars $S$ was due to complementation of ars $S$ rather than the inactivation of $r d x A$. A hyper-adherent phenotype similar to that of J99ars $S:: \mathrm{km}$ was also seen in a J99-arsS::cat strain, demonstrating that independently generated ars $S$-null strains possess the same phenotype (data not shown). In contrast, there was no increase in adherence of $H$. pylori strain 26695-arsS::cat relative to wild-type 26695 $\left[2.5( \pm 0.3) \times 10^{5}\right.$ vs $\left.3.9( \pm 0.5) \times 10^{5}, P=0.3, n=2\right]$. There was also a trend towards lower basal adherence by wildtype strain 26695 than by J99, potentially due to the lack of SabA-mediated binding in 26695. Further studies will be necessary to elucidate what other factors, such as relative BabA expression, may impact the degree of adherence to AGS cells.

These results establish the functional significance of two distinct mechanisms regulating the expression of the SabA adhesin. The elimination of ArsS in $\mathrm{J} 99$ led to a greater than 10-fold increase in adherence that was SabAdependent, importantly demonstrating that the transcriptional regulation of $s a b A$ by the ArsRS TCST results in functional changes in $H$. pylori adherence to gastric epithelial cells. However, this elevated binding upon disruption of arsS only occurred in strain J99, which contains an in-frame sabA allele, but not in strain 26695, which possesses a predominantly out-of-frame sabA locus.

Persistent $H$. pylori colonization requires continual adaptation to variations in the gastric microenvironments it inhabits, and to robust host immune and inflammatory responses. Due to the fact that the $H$. pylori genome contains relatively few conserved transcriptional regulators, alternative mechanisms of gene regulation, such as variation in repetitive DNA sequences, play an important role in generating genetic diversity in H. pylori (Aras et al., 2003; Mrazek et al., 2007). The present study demonstrates an example of how TCST-mediated regulation and phase variation combine to regulate the transcription of $s a b A$, and the subsequent ability of $H$. pylori to bind sialyl-Le ${ }^{\mathrm{x}}$ displayed on gastric epithelial cells. The combination of transcriptional regulation of the $s a b A$ locus by the ArsRS TCST system, and the generation of subpopulations harbouring alternate $s a b A$ alleles by slipped-strand mispairing during chromosomal replication, could permit $H$. pylori to rapidly adapt to varying microenvironments or host immune responses. The existence of multiple means by which the expression of SabA is controlled in H. pylori suggests that precise regulation of this adhesin may be crucial to the virulence of this important pathogen.

\section{ACKNOWLEDGEMENTS}

This work was supported by grants from the National Institutes of Health (AI53062), the Commonwealth Health Research Board (122004), and the Thomas F. and Kate Miller Jeffress Memorial Trust (J602) to M.H.F., as well as National Institutes of Health grants DK 58587, CA77955 and DK 73902 to R.M.P. This research was also supported in part by a Howard Hughes Medical Institute grant to the College of William and Mary through the Undergraduate Biological Sciences Education Program, the Corporate Partners Travel Grant program of the American Society for Microbiology, and the College of William and Mary. The authors wish to thank Dr Alison Criss (Northwestern University, Chicago, IL) for advice on cell-adhesion assays.

\section{REFERENCES}

Acheson, D. W. \& Luccioli, S. (2004). Microbial-gut interactions in health and disease. Mucosal immune responses. Best Pract Res Clin Gastroenterol 18, 387-404.

Alm, R. A., Ling, L. S., Moir, D. T., King, B. L., Brown, E. D., Doig, P. C., Smith, D. R., Noonan, B., Guild, B. C. \& other authors (1999). Genomic-sequence comparison of two unrelated isolates of the human gastric pathogen Helicobacter pylori. Nature 397, 176-180.

Alper, J. (2001). Searching for medicine's sweet spot. Science 291, 2338-2343.

Aras, R. A., Kang, J., Tschumi, A. I., Harasaki, Y. \& Blaser, M. J. (2003). Extensive repetitive DNA facilitates prokaryotic genome plasticity. Proc Natl Acad Sci U S A 100, 13579-13584.

Aspholm, M., Kalia, A., Ruhl, S., Schedin, S., Arnqvist, A., Linden, S., Sjostrom, R., Gerhard, M., Semino-Mora, C. \& other authors (2006). Helicobacter pylori adhesion to carbohydrates. Methods Enzymol 417, 293-339. 
Beier, D. \& Frank, R. (2000). Molecular characterization of twocomponent systems of Helicobacter pylori. J Bacteriol 182, 2068-2076.

Bjorkholm, B. \& Salama, N. R. (2003). Genomics of Helicobacter 2003. Helicobacter 8 (Suppl. 1), 1-7.

Blaser, M. J. \& Berg, D. E. (2001). Helicobacter pylori genetic diversity and risk of human disease. J Clin Invest 107, 767-773.

Boren, T., Falk, P., Roth, K. A., Larson, G. \& Normark, S. (1993). Attachment of Helicobacter pylori to human gastric epithelium mediated by blood group antigens. Science 262, 1892-1895.

Bury-Mone, S., Thiberge, J. M., Contreras, M., Maitournam, A., Labigne, A. \& De Reuse, H. (2004). Responsiveness to acidity via metal ion regulators mediates virulence in the gastric pathogen Helicobacter pylori. Mol Microbiol 53, 623-638.

de Jonge, R., Pot, R. G., Loffeld, R. J., van Vliet, A. H., Kuipers, E. J. \& Kusters, J. G. (2004). The functional status of the Helicobacter pylori $s a b B$ adhesin gene as a putative marker for disease outcome. Helicobacter 9, 158-164.

de Vries, N., Duinsbergen, D., Kuipers, E. J., Pot, R. G., Wiesenekker, P., Penn, C. W., van Vliet, A. H., Vandenbroucke-Grauls, C. M. \& Kusters, J. G. (2002). Transcriptional phase variation of a type III restrictionmodification system in Helicobacter pylori. J Bacteriol 184, 6615-6623.

Dietz, P., Gerlach, G. \& Beier, D. (2002). Identification of target genes regulated by the two-component system HP166-HP165 of Helicobacter pylori. J Bacteriol 184, 350-362.

Forsyth, M. H., Cao, P., Garcia, P. P., Hall, J. D. \& Cover, T. L. (2002). Genome-wide transcriptional profiling in a histidine kinase mutant of Helicobacter pylori identifies members of a regulon. J Bacteriol 184, 4630-4635.

Gerhard, M., Lehn, N., Neumayer, N., Boren, T., Rad, R., Schepp, W., Miehlke, S., Classen, M. \& Prinz, C. (1999). Clinical relevance of the Helicobacter pylori gene for blood-group antigen-binding adhesin. Proc Natl Acad Sci U S A 96, 12778-12783.

Ilver, D., Arnqvist, A., Ogren, J., Frick, I. M., Kersulyte, D., Incecik, E. T., Berg, D. E., Covacci, A., Engstrand, L. \& other authors (1998). Helicobacter pylori adhesin binding fucosylated histo-blood group antigens revealed by retagging. Science 279, 373-377.

Israel, D. A., Salama, N., Krishna, U., Rieger, U. M., Atherton, J. C., Falkow, S. \& Peek, R. M., Jr (2001). Helicobacter pylori genetic diversity within the gastric niche of a single human host. Proc Natl Acad Sci U S A 98, 14625-14630.

Kuipers, E. J., Israel, D. A., Kusters, J. G., Gerrits, M. M., Weel, J., van Der Ende, A., van Der Hulst, R. W., Wirth, H. P., Hook-Nikanne, J. \& other authors (2000). Quasispecies development of Helicobacter pylori observed in paired isolates obtained years apart from the same host. J Infect Dis 181, 273-282.

Kusters, J. G., van Vliet, A. H. \& Kuipers, E. J. (2006). Pathogenesis of Helicobacter pylori infection. Clin Microbiol Rev 19, 449-490.

Lehours, P., Menard, A., Dupouy, S., Bergey, B., Richy, F., Zerbib, F., Ruskone-Fourmestraux, A., Delchier, J. C. \& Megraud, F. (2004). Evaluation of the association of nine Helicobacter pylori virulence factors with strains involved in low-grade gastric mucosa-associated lymphoid tissue lymphoma. Infect Immun 72, 880-888.

Linden, S., Boren, T., Dubois, A. \& Carlstedt, I. (2004). Rhesus monkey gastric mucins: oligomeric structure, glycoforms and Helicobacter pylori binding. Biochem J 379, 765-775.

Livak, K. J. \& Schmittgen, T. D. (2001). Analysis of relative gene expression data using real-time quantitative PCR and the $2^{-\triangle \Delta C T}$ method. Methods 25, 402-408.

Loh, J. T. \& Cover, T. L. (2006). Requirement of histidine kinases HP0165 and HP1364 for acid resistance in Helicobacter pylori. Infect Immun 74, 3052-3059.
Madrid, J. F., Ballesta, J., Castells, M. T. \& Hernandez, F. (1990). Glycoconjugate distribution in the human fundic mucosa revealed by lectin- and glycoprotein-gold cytochemistry. Histochemistry 95, 179-187.

Mahdavi, J., Sonden, B., Hurtig, M., Olfat, F. O., Forsberg, L., Roche, N., Angstrom, J., Larsson, T., Teneberg, S. \& other authors (2002). Helicobacter pylori SabA adhesin in persistent infection and chronic inflammation. Science 297, 573-578.

Merrell, D. S. \& Falkow, S. (2004). Frontal and stealth attack strategies in microbial pathogenesis. Nature 430, 250-256.

Merrell, D. S., Goodrich, M. L., Otto, G., Tompkins, L. S. \& Falkow, S. (2003). pH-regulated gene expression of the gastric pathogen Helicobacter pylori. Infect Immun 71, 3529-3539.

Mrazek, J., Guo, X. \& Shah, A. (2007). Simple sequence repeats in prokaryotic genomes. Proc Natl Acad Sci U S A 104, 8472-8477.

Odenbreit, S. (2005). Adherence properties of Helicobacter pylori: impact on pathogenesis and adaptation to the host. Int $\mathrm{J} \mathrm{Med}$ Microbiol 295, 317-324.

Panthel, K., Dietz, P., Haas, R. \& Beier, D. (2003). Two-component systems of Helicobacter pylori contribute to virulence in a mouse infection model. Infect Immun 71, 5381-5385.

Pflock, M., Dietz, P., Schar, J. \& Beier, D. (2004). Genetic evidence for histidine kinase HP165 being an acid sensor of Helicobacter pylori. FEMS Microbiol Lett 234, 51-61.

Pflock, M., Kennard, S., Delany, I., Scarlato, V. \& Beier, D. (2005). Acid-induced activation of the urease promoters is mediated directly by the ArsRS two-component system of Helicobacter pylori. Infect Immun 73, 6437-6445.

Roche, N., Angstrom, J., Hurtig, M., Larsson, T., Boren, T. \& Teneberg, S. (2004). Helicobacter pylori and complex gangliosides. Infect Immun 72, 1519-1529.

Rubinsztein-Dunlop, S., Guy, B., Lissolo, L. \& Fischer, H. (2005). Identification of two new Helicobacter pylori surface proteins involved in attachment to epithelial cell lines. J Med Microbiol 54, 427-434.

Sachs, G., Kraut, J. A., Wen, Y., Feng, J. \& Scott, D. R. (2006). Urea transport in bacteria: acid acclimation by gastric Helicobacter spp. $J$ Membr Biol 212, 71-82.

Salaun, L., Linz, B., Suerbaum, S. \& Saunders, N. J. (2004). The diversity within an expanded and redefined repertoire of phasevariable genes in Helicobacter pylori. Microbiology 150, 817-830.

Saunders, N. J., Peden, J. F., Hood, D. W. \& Moxon, E. R. (1998). Simple sequence repeats in the Helicobacter pylori genome. Mol Microbiol 27, 1091-1098.

Schar, J., Sickmann, A. \& Beier, D. (2005). Phosphorylationindependent activity of atypical response regulators of Helicobacter pylori. J Bacteriol 187, 3100-3109.

Sheu, B. S., Odenbreit, S., Hung, K. H., Liu, C. P., Sheu, S. M., Yang, H. B. \& Wu, J. J. (2006). Interaction between host gastric sialyl-Lewis X and $H$. pylori SabA enhances $H$. pylori density in patients lacking gastric Lewis B antigen. Am J Gastroenterol 101, 36-44.

Stock, A. M., Robinson, V. L. \& Goudreau, P. N. (2000). Twocomponent signal transduction. Annu Rev Biochem 69, 183-215.

Tomb, J. F., White, O., Kerlavage, A. R., Clayton, R. A., Sutton, G. G., Fleischmann, R. D., Ketchum, K. A., Klenk, H. P., Gill, S. \& other authors (1997). The complete genome sequence of the gastric pathogen Helicobacter pylori. Nature 388, 539-547.

van der Woude, M. W. \& Baumler, A. J. (2004). Phase and antigenic variation in bacteria. Clin Microbiol Rev 17, 581-611.

Yamaoka, Y., Kita, M., Kodama, T., Imamura, S., Ohno, T., Sawai, N., Ishimaru, A., Imanishi, J. \& Graham, D. Y. (2002). Helicobacter pylori 
infection in mice: role of outer membrane proteins in colonization and inflammation. Gastroenterology 123, 1992-2004.

Yamaoka, Y., Ojo, O., Fujimoto, S., Odenbreit, S., Haas, R., Gutierrez, O., El-Zimaity, H. M., Reddy, R., Arnqvist, A. \& other authors (2006). Helicobacter pylori outer membrane proteins and gastroduodenal disease. Gut 55, 775-781.

Edited by: N. J. High 